Original Research Paper

\title{
Synthesis of Graphene Oxide via Liquid Exfoliation Using Self-Custom-Made Tweeter Piezoelectric Ultrasound Generator and Assisted by Surfactant from Commercial Detergent
}

\author{
${ }^{1}$ Achmad Ainul Fikri, ${ }^{1}$ Aminah Nur Aisyah, ${ }^{2}$ Suhufa Alfarisa and ${ }^{1}$ Wipsar Sunu Brams Dwandaru \\ ${ }^{1}$ Department of Physics Education, Universitas Negeri Yogyakarta, Yogyakarta, Indonesia \\ ${ }^{2}$ Department of Physics, Universitas PGRI Palembang, Palembang, Indonesia
}

Article history

Received: 17-06-2016

Revised: 23-09-2016

Accepted: 02-11-2016

Corresponding Author: W.S.B. Dwandaru

Physics Education Department, Universitas Negeri Yogyakarta, Yogyakarta, Indonesia Email: wipsarian@yahoo.com

\begin{abstract}
Rapid progress in nanotechnology requires the production of novel nanomaterials. This study aims to synthesize Graphene Oxide (GO) via the Liquid Exfoliation (LE) method using a self-custom-made tweeter piezoelectric system as the ultrasound generator. Linear Alkylbenzena Sulfonate (LAS) surfactant contained in commercial detergent is used to assist the exfoliation process. This synthesis method is simple, inexpensive and may produce GO in a large quantity. Furthermore, the effect of the sonication time on the synthesis of GO is studied. The UV-Vis spectrophotometer and Scanning Electron Microscope (SEM) are used to characterize the synthesis results. UV-Vis analysis shows that the longer the sonication time spent, the thinner the GO layers produced. Moreover, images from SEM show that the surface morphology of the GO forms transparent layers which are stacked together on top of each other.
\end{abstract}

Keywords: Graphene Oxide, Liquid Exfoliation, Surfactant, Tweeter Piezoelectric Ultrasound Generator

\section{Introduction}

Graphene is known as the thinnest and strongest material in the world today which is formed from a single layer of hexagonal honeycomb-shaped carbon atom bounded by covalent bonds (Wang et al., 2013; RSAS, 2010). Many carbon-based materials, such as graphite, carbon nanotube and fullerene are formed by graphene as their basic structure (Basu and Bhattacharyya, 2012). Graphene was first synthesized by Novoselov et al. (2004) using the Mechanical Exfoliation (ME) method involving the application of a sticky tape.

Graphene has been an interesting material to be investigated because of its superior properties such as high electron mobility, high conductivity, high thermal conductivity, good optical transparency and high Young's modulus (Terrones et al., 2010). Furthermore, graphene can be applied in various fields such as electronics industry for the manufacture of supercapacitors and transistors (Li et al., 2013; Zhao et al., 2009; El-Kady and Kaner, 2013).

The excitement in graphene leads to the finding of large scale production of graphene layers for the aforementioned application purposes without losing the above novel properties. One way of achieving this is by graphite oxidation via strong oxidizing agents such that graphite oxide or GO is obtained (Dreyer et al., 2009). Although GO is considered as a forerunner in obtaining graphene, the material itself is being extensively studied for its various uses, such as in biomedical (Chung et al., 2013) and optical (Loh et al., 2010) applications, antibacterial material especially integrated with silver nanoparticles and bacterial cellulose (Zhang et al., 2011; Tang et al., 2013; Shao et al., 2015), bio-sensors (Shao et al., 2010; Liu et al., 2010), hydrogen storage (Wang et al., 2009; Tylianakis et al., 2010; Kim et al., 2012) and filtration membranes (Joshi et al., 2014; Xu et al., 2013).

Many methods has been used in synthesizing graphene or GO. These methods are quite established in various literatures, e.g., ME method (Yi and Shen, 2015), Chemical Vapor Deposition (CVD) (Obraztsov, 2009; Chen et al., 2011), epitaxial growth (Sutter et al., 2008; Yang et al., 2013) and Hummer's method (Reina et al., 2009; Marcano et al., 2010). Another synthesis 
method called reduction of Graphene Oxide (rGO) (Stankovich et al., 2007; Pei and Cheng, 2012) is promising due to the large production of graphene, although this method can reduce the electronic performance of graphene (Risley, 2013).

The LE method offers an easier, more efficient and simpler way to synthesize graphene in a large quantity and a good quality (Wang et al., 2016; Hernandez et al., 2008). In this way, surfactant is utilized to assist the exfoliation process of graphene layers from graphite material (Murat et al., 2012). The LE method can produce a stable and good quality of graphene in spite of the defects brought by surfactant (Park and Rouff, 2009; Li et al., 2008). Many researchers then adopted and developed this method in order to optimize the production and quality of graphene or GO. For example, exfoliation of graphite material may be conducted by combining the LE method with electrolysis process (Tang et al., 2012), using kitchen blender (Yi and Shen, 2014), or even sonication (Khan et al., 2010; Bang and Sulick, 2010; Durge et al., 2014).

This study reports the synthesis of GO using the LE method by applying a self-custom-made ultrasound generator consisting of tweeter piezoelectric probes as the ultrasound sources. These probes are used because they are easy to obtain and quite cheap, hence suggesting a reduction in the cost of producing GO. Moreover, they may produce audible and ultrasound frequency range of sound waves which are important in separating graphene layers. Thinner layers of graphene will separate from the thicker ones which are still in the form of cloudlike graphite. The effect of sonication can be directly observed by the increase of the temperature and the change of the solution color into grayish black. Although the use of ultrasound for the exfoliation of graphite or graphite oxide in the LE method is already available in literatures, however, to the knowledge of the authors, the design and use of the ultrasound generator from tweeter piezoelectric probes in this study has not been conducted. Here, the effect of sonication time of the tweeter piezoelectric system towards the synthesis of GO is studied using the UVVis spectrophotometer and SEM images.

Meanwhile, LAS surfactants contained in commercial detergents are used to assist the exfoliation process. Surfactants weaken the Van der Waals bond between graphene layers in the graphite material, which is then followed by the separation of graphene layers due to vibrations during the sonication process. By using inexpensive commercial detergents which contain LAS surfactants, the cost of producing GO may be further reduced.

\section{Experimental Method}

The main materials employed in this study are (i) graphite powder from Faber-Castell 2B commercial pencil, (ii) commercial detergent containing $20 \%$ LAS surfactants and (iii) distilled water. Graphite and detergent powders are illustrated in Fig. 1 (below-right picture). The main equipments utilized are an audio generator (CSi/SPECO SS-1), an amplifier (Uchida TA2MS) and tweeter piezoelectric probes (Fig. 1 [top and below-left pictures]), which constitute the tweeter piezoelectric ultrasound generator. The ultrasound source apparatus (Fig. 1 top picture) is constructed from a second-hand (used) drinking bottle mounted on a large wooden board as the main pole supporting three piezoelectric probe assemblies which are hanging via flexible cables. Each assembly consists further of three probes (Fig. 1 below-left picture) with each probe attached to the side of a triangle-shaped wooden thinboard.

In the sonication process, each of the assembly is then submerged into the liquid solution sample providing vibrations inside the liquid sample in three directions (Fig. 2 below). Moreover, each of the probes on each assembly may be turned on or off using switches on the large board, such that the number of probes sonicated in the liquid may be varied. On the other hand, the frequency of sound wave in audible and ultrasound ranges may be varied using the audio generator. The results of these two aforementioned variations are being reported elsewhere.

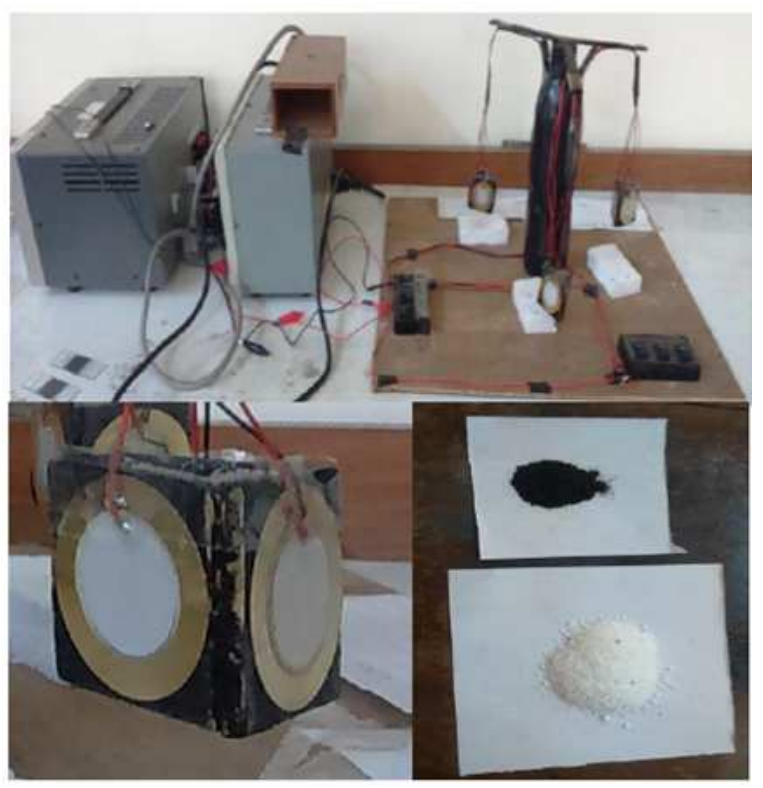

Fig. 1. Tweeter piezoelectric ultrasound generator, consisting of an audio generator, an amplifier and an ultrasound source apparatus (top picture), a tweeter piezoelectric probe assembly (below-left) and graphite (black) and detergent (white) powders [below-right] 


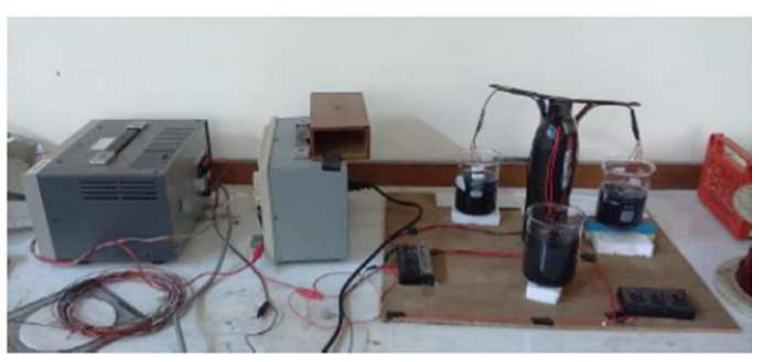

Fig. 2. Sonication process of the solution samples

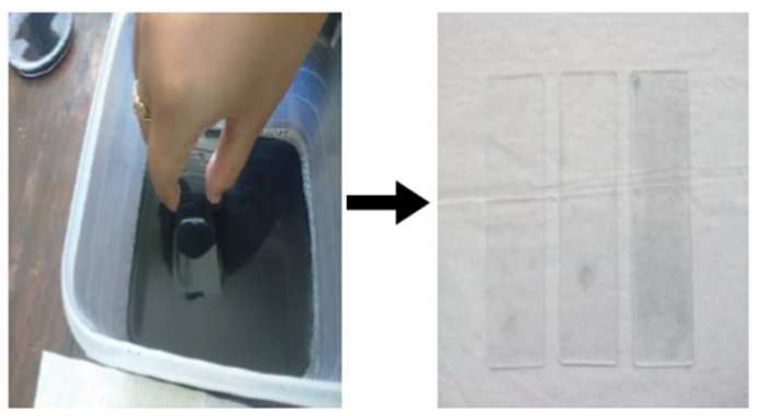

Fig. 3. Dip coating process of the sample from a liquid phase.

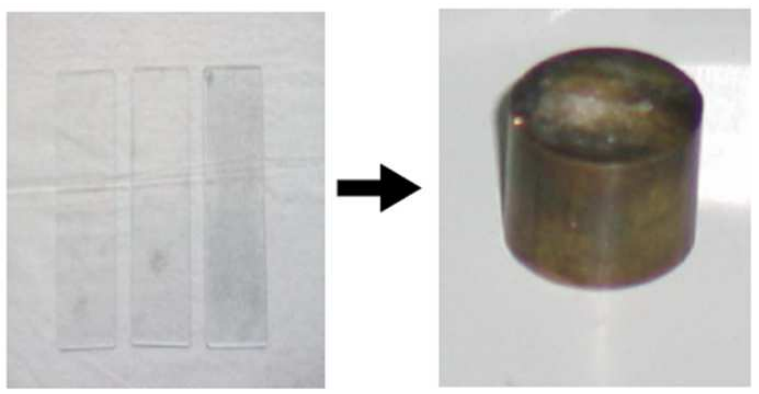

Fig. 4. The solidified sample is moved onto a speciment

The procedures of the experiment in this study are given as follows. Graphite powder as much as 0.5 gram is mixed into a surfactant solution with an amount of $0.025 \mathrm{~g} \mathrm{~mL}^{-1}$. The surfactant solution is obtained by mixing $200 \mathrm{~mL}$ of distilled water with 5 grams of commercial detergent. The prepared graphite solution is then separated into four beaker glasses. Each one of the beaker glasses is sonicated for 1,3 and $5 \mathrm{~h}$ (Fig. 2 above). The last beaker glass is left without sonication process. All of the solutions are then left overnight.

A UV-Visible spectroscopy is conducted upon the supernatant of the solution which has been left overnight. Characterization of the samples is done using a UV-Vis spectrophotometer (Shimadzu UV-2450) in the range of 200 to $700 \mathrm{~nm}$. Moreover, some of the supernatant solution is transferred onto a glass substrate via a dip coating process. Figure 3 shows the dip coating process of the supernatant onto a glass substrate. The substrate is then annealed in an oven for $10 \mathrm{~min}$ at $150^{\circ} \mathrm{C}$. Subsequently, SEM (JEOL JSM T300) analysis is conducted at $30 \mathrm{kV}$ for the sample on the substrate (Fig. 4). SEM is performed only for the sample with $5 \mathrm{~h}$ sonication time.

\section{Results and Discussion}

\section{Liquid Solution Sample after Sonication}

The solution sample after sonication may be observed in Fig. 5a. A temperature increase of the solution as a result of sonication is detected as the beaker glass becomes warm (upon touching it) during and right after the sonication process. It may be observed that the color of the solution after the sonication is blue on top of the solution and becomes darker going down to the bottom. Figure $5 \mathrm{~b}$ shows the solution after it is being left overnight. There is obviously a change in the color of the solution. After being left overnight, the solution becomes grey. There are also dark sediments on the bottom of the solution.

\section{UV-Vis Characterization}

Figure 6 shows the UV-Vis result of the samples with $0.025 \mathrm{~g} \mathrm{~mL}^{-1}$ of surfactant and varying sonication time, viz. (in hour): $0,1,3$ and 5 . The graph is obtained by subtracting the UV-Vis absorbance data of the solution from the corresponding absorbance data of the pure surfactant after sonication. The diamond (blue), square (red), triangle (green) and crossed (purple) point data are the absorbance of the sample solutions with $0,1,3$ and 5 $\mathrm{h}$ of sonication time, respectively.

The graphs in Fig. 6 show different absorbance peaks for each sonication time. For 1,3 and $5 \mathrm{~h}$ of sonication time, there are two absorbance peaks which occur at similar wavelengths, i.e.: 270 and $340 \mathrm{~nm}$. However, the absorbance peaks take place in different absorbance values. The absorbance peaks on 270 and $340 \mathrm{~nm}$ are characteristics of GO or multilayered graphene (Murat et al., 2012). This is of course different from the absorbance characteristics of the solution without sonication (diamond [blue] data). Without sonication $(0 \mathrm{~h})$, the peak on $270 \mathrm{~nm}$ is less pronounced, which indicates that the solution may still consist of graphite or graphite oxide.

It may also be observed that as the sonication time spent gets longer, the value of the absorbance peaks decreases. This indicates that the longer the sonication time spent, the thinner the layers of the GO obtained. Moreover, this means that the solution with five hours of sonication time gives the thinnest layers of multilayered graphene. This is evidenced from the lowest peaks of the absorbance at 270 and $340 \mathrm{~nm}$ of the crossed (purple) data. This is because the layers of the multilayered graphene undergo more exfoliations as it is exposed to longer time of ultrasound vibrations. 


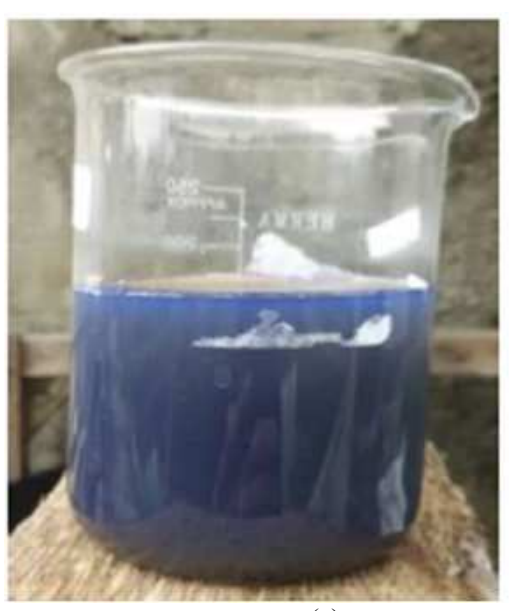

(a)

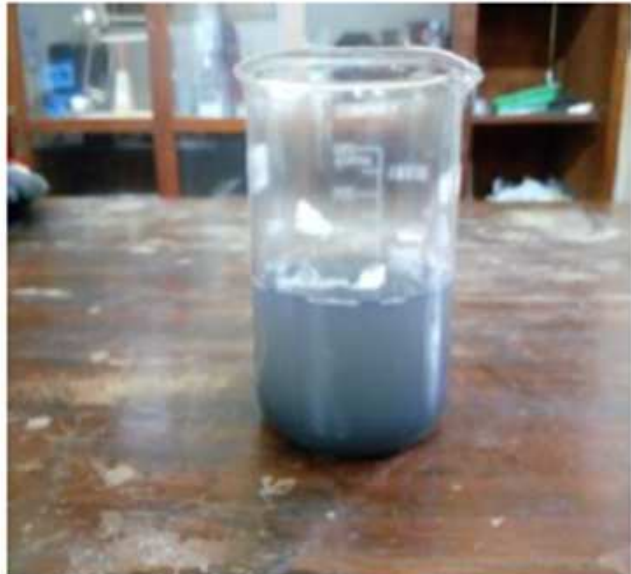

(b)

Fig. 5. (a) The solution sample after being sonificated and (b) the solution sample after being left overnight

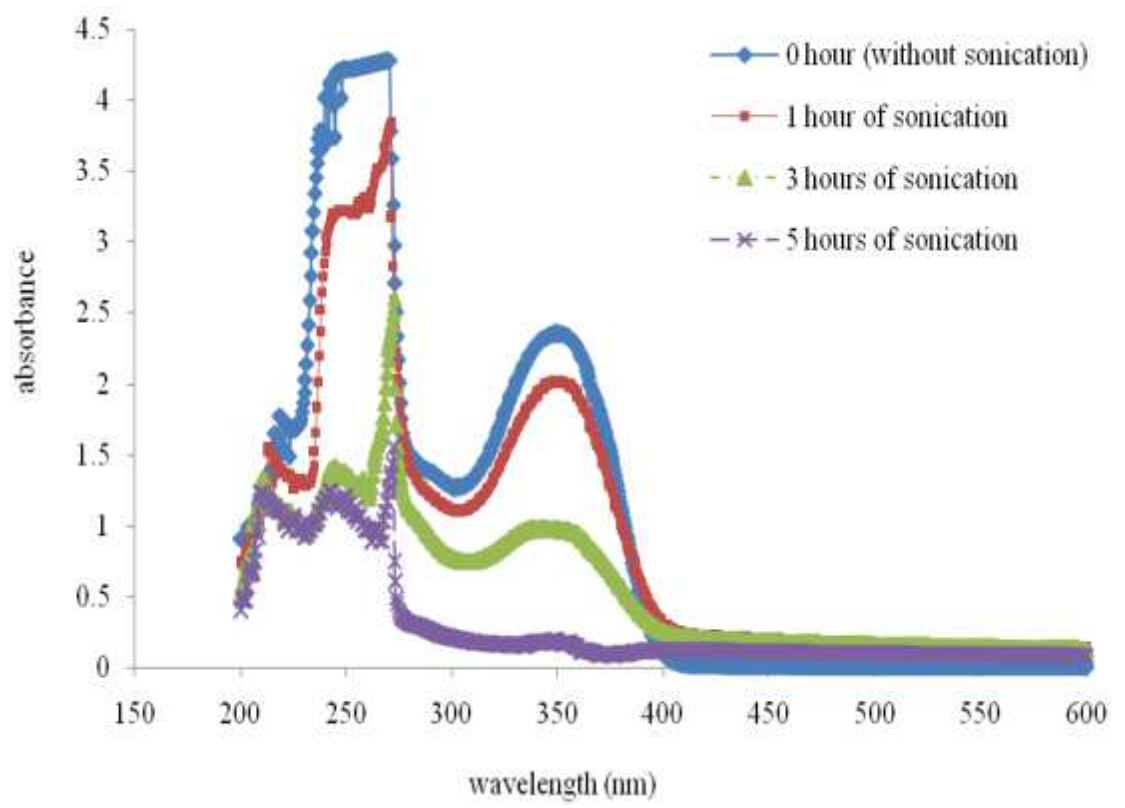

Fig. 6. UV-Vis results of the solution samples with $0.025 \mathrm{~g} \mathrm{~mL}^{-1}$ surfactant and varying sonication time of $0,1,3$ and $5 \mathrm{~h}$

\section{SEM Results}

The SEM analysis is conducted to determine the surface morphology of the material produced. The solution (supernatant) is initially solidified before being characterized using SEM. The supernatant part is solidified by deep coating the glass with the liquid sample solution.

From the UV-Vis result it is obtained that the sample produced with five hours of sonication time gives the most optimize GO's performance (thinnest layers of multi-layered graphene). Therefore, further analysis using SEM is only conducted on that sample. Figure 7 presents the surface morphology of the solution that undergoes sonication for five hours. Figure $7 \mathrm{a}$ displays the solidified material distribution of the sample with $100 \mathrm{X}$ magnification. It shows an island of materials on the left-bottom part of the figure. Additionally, smaller materials are scattered throughout the figure with widths ranging from 2.5 to 28 microns. Figure $7 \mathrm{~b}$ shows cloudlike graphene oxides with $1000 \mathrm{X}$ magnification. Layering may also be observed although it is less obvious. Figure 7c illustrates cloud-like graphene oxides with further magnification $(2000 \mathrm{X})$ which clearly shows stacking of graphene materials on top of each other. This illustrates that GO is produced in this study. 

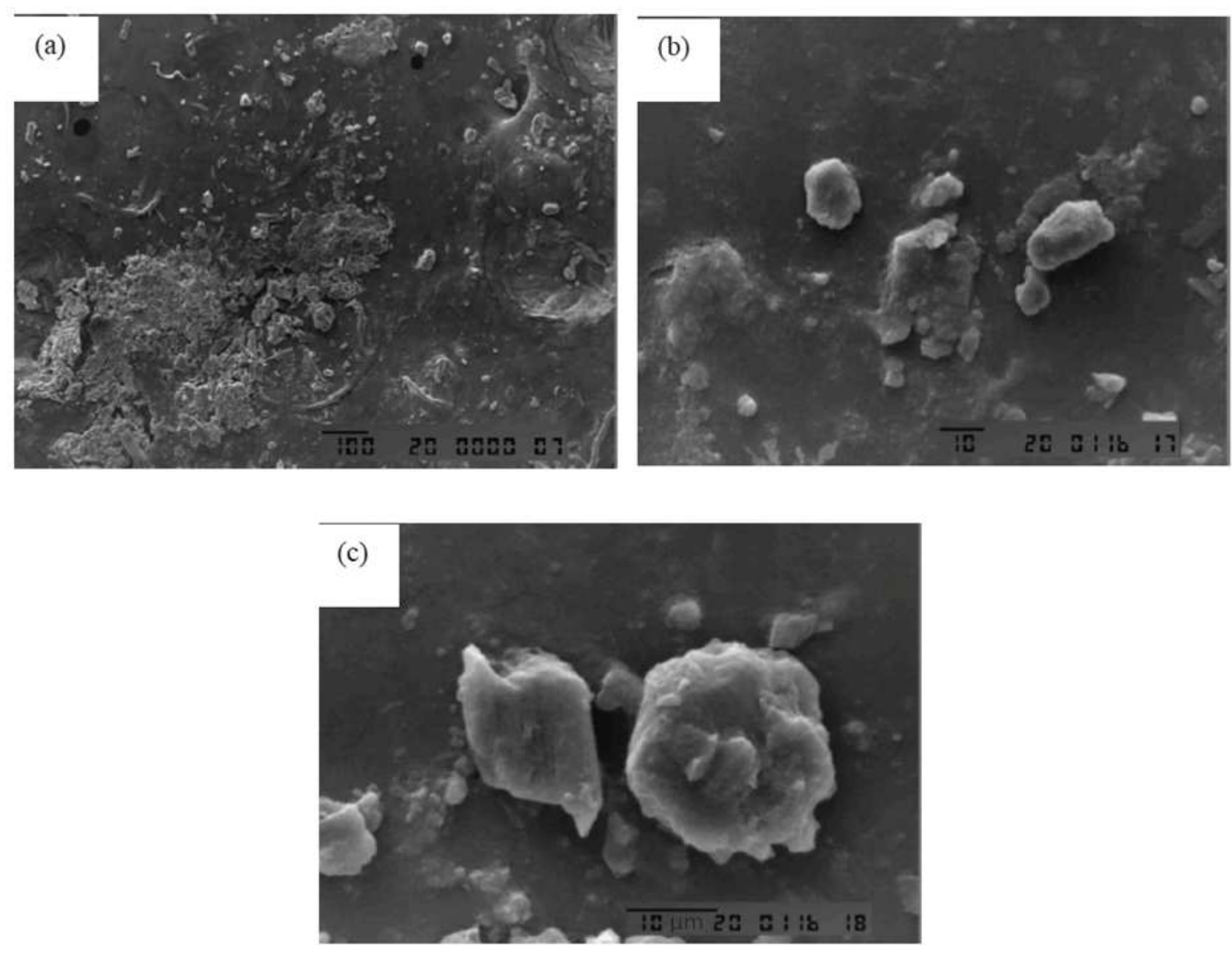

Fig. 7. SEM results for five hours of sonication time taken at different positions on the sample with (a) 100X, (b) $1000 \mathrm{X}$ and (c) 2000X magnifications

\section{Conclusion}

Synthesis of GO with a simple and inexpensive method of LE has been conducted. Here, the production of GO uses a self-custom-made tweeter piezoelectric ultrasound generator and assisted by LAS surfactant from commercial detergent. The UV$\mathrm{Vis}$ analysis shows that the longer the sonication time spent, the thinner the GO layers produced. SEM results illustrate that the surface morphology of the GO layers consist of transparent layers of graphenes stacked together on top of each other. This study contributes to the many methods in synthesizing GO, especially using simple and inexpensive materials and tools.

\section{Acknowledgement}

The authors would also like to express gratitude to Bambang Sugeng, M.Pd., Ph.D for reviewing the language of this article.

\section{Funding Information}

This study has been supported by the Faculty of Mathematics and Natural Science of Universitas Negeri Yogyakarta under the funding of DANA DIPA Fakultas MIPA 2016.

\section{Author's Contributions}

Achmad Ainul Fikri and Aminah Nur Aisyah: Gave significant contribution in the preparation of this article. They conducted the experiments and data collecting, including assembling the self-custom-made tweeter piezoelectric ultrasound generator. Produced the initial draft of the article.

Suhufa Alfarisa and Wipsar Sunu Brams Dwandaru: Were the supervisors who provided the main idea and oversaw the overall research project, including making sure that the experiments were conducted correctly as planned. Reviewed and finalized the draft of the article before it is being submitted. 


\section{References}

Bang, J.H. and K.S. Sulick, 2010. Applications of ultrasound to the synthesis of nanostructured materials. Adv. Mater., 22: 1039-1059.

DOI: 10.1002/adma.200904093

Basu, S. and P. Bhattacharyya, 2012. Recent developments on graphene and graphene oxide based solid state gas sensors. Sensor Actuators B, 173: 1-21 DOI: 10.1016/j.snb.2012.07.092

Chen, Z., W. Ren, L. Gao, B. Liu and S. Pei et al., 2011. Three-dimensional flexible and conductive interconnected graphene networks grown by chemical vapour deposition. Nat. Mater., 10: 424-428. DOI: $10.1038 /$ nmat3001

Chung, C., Y.K. Kim, D. Shin, S.R. Ryoo and B.H. Hong et al., 2013. Biomedical applications of graphene and graphene oxide. Acc. Chem. Res., 46: 2211-2224 DOI: 10.1021/ar300159f

Dreyer, D.R., S. Park, C.W. Bielawski and R.S. Rouff, 2010. The chemistry of graphene oxide. Chem. Soc. Rev., 39: 228-240 DOI: 10.1039/b917103g

Durge, R., R.V. Kshirsagar and P. Tambe, 2014. Effect of sonication energy on the yield of graphene nanosheets by liquid-phase exfoliation of graphene. Proc. Eng., 97: 1457-1465.

DOI: $10.1016 /$ j.proeng.2014.12.429

El-Kady, M.F. and R.B. Kaner, 2013. Scalable fabrication of high-power graphene microsupercapacitors for flexible on-chip energy storage. Nat. Commun., 4: 1475-1475.

DOI: $10.1038 /$ ncomms 2446

Hernandez, Y., V. Nicolosi, M. Lotya, F.M. Blighe and Z. Sun et al., 2008. Highyield production of graphene by liquid-phase exfoliation of graphite. Nat. Nanotechnol., 3: 563-568.

DOI: $10.1038 /$ nnano.2008.215

Joshi, R.K., P. Carbone, F.C. Wang, V.G. Kravets and Y. Su et al., 2014. Precise and ultrafast molecular sieving through graphene oxide membranes. Science, 343: 752-754

DOI: $10.1126 /$ science. 1245711

Khan, U., A. O’Neill, M. Lotya, S. De and J.N. Coleman, 2010. High-concentration solvent exfoliation of graphene. Small, 6: 864-871. DOI: $10.1002 /$ smll.200902066

Kim, B.H., W.G. Hong, H.Y. Yu, Y.K. Han and S.M. Lee et al., 2012. Thermally modulated multilayered graphene oxide for hydrogen storage. Phys. Chem. Chem. Phys., 14: 1480-1484. DOI: $10.1039 / \mathrm{C} 2 \mathrm{CP} 23683 \mathrm{D}$

Li, D., M.B. Müller and S. Gilje, 2008. Processable aqueous dispersions of graphene nanosheets. Nat. Nanotechnol., 3: 101-105.

DOI: $10.1038 /$ nnano.2007.451
Li, X., T. Zhao, Q. Chen, P. Li and K. Wang et al., 2013. Flexible all solid-state supercapacitors based on chemical vapor deposition derived graphene fibers. Phys. Chem. Chem. Phys., 15: 17752-17757. DOI: $10.1039 /$ C3CP52908H

Liu, Y., D. Yu, C. Zheng, Z. Miao and L. Dai, 2010. Biocompatible graphene-oxide based glucose biosensors. Langmuir, 26: 6158-6160. DOI: $10.1021 /$ la100886x

Loh, K.P., Q. Bao, G. Eda and M. Chhowalla, 2010. Graphene oxide as a chemically tunable platform for optical applications. Nat. Chem., 2: 1015-1024. DOI: $10.1038 /$ nchem.907

Marcano, D.C., D.V. Kosynkin, J.M. Berlin, A. Sinitskii and Z. Sun et al., 2010. Improved synthesis of graphene oxide. ACS Nano, 4: 4806-4814. DOI: $10.1021 / \mathrm{nn} 1006368$

Murat, A., J.J. Segura, J. Oro-Sole and N. Casan-Pastor, 2012. The Synthesis of graphene sheets with controlled thickness and order using surfactantassisted electrochemical processes. Carbon, 50: 142-152.

DOI: $10.1016 /$ j.carbon.2011.07.064

Novoselov, K.S., A.K. Geim, S.V. Morozov, D. Jiang and Y. Zhang et al., 2004. Electric field effect in atomically thin carbon films. Science, 306: 666-669. DOI: $10.1126 /$ science. 1102896

Obraztsov, A.N., 2009. Chemical vapor deposition: making grahene on a large scale. Nat. Nanotechnol., 4: 212-213. DOI: 10.1038/nnano.2009.67

Park, S. and R.S. Rouff, 2009. Chemical methods for the production of graphenes. Nat. Nanotechnol., 4: 217-224. DOI: $10.1038 /$ nnano.2009.58

Pei, S. and H.M. Cheng, 2012. The reduction of grahene oxide. Carbon, 50: 3210-3228. DOI: $10.1016 /$ j.carbon.2011.11.010

Reina, A., X. Jia, J. Ho, D. Nezich and H. Son et al., 2009. Large area, few-layer graphene films on arbitrary substrates by chemical vapor deposition. Nano Lett., 9: 30-35 DOI: 10.1021/n1801827v

Risley, M.J., 2013. Surfactant-assisted solution exfoliation and processing of graphite and graphene. M.Sc. Thesis. School of Chemical and Biomolecular Engineering, Georgia Institute of Technology, Georgia.

RSAS, 2010. Graphene. Kungliga Vetenskapsakademien, The Royal Swedish Academy of Sciences, Press Release.

Shao, W., H. Liu, X. Liu, S. Wang and R. Zhang, 2015. Anti-bacterial performances and biocompatibility of bacterial cellulose/graphene oxide composites. RSC Adv., 5: 4795-4803. DOI: $10.1039 /$ C4RA13057J 
Shao, Y., J. Wang, H. Wu, J. Liu and I.A. Aksay et al., 2010. Graphene based electrochemical sensors and biosensors: A review. Electroanalysis, 22: 1027-1036. DOI: 10.1002/elan.200900571

Stankovich, S., D.A. Dikin, R.D. Piner, K.A. Kohlhaas and A. Kleinhammes et al., 2007. Synthesis of graphenebased nanosheets via chemical reduction of exfoliated graphite oxide. Carbon, 45: 1558-1565 DOI: 10.1016/j.carbon.2007.02.034

Sutter, P.W., J.I. Flege and E.A. Sutter, 2008. Epitaxial graphene on ruthenium. Nat. Mater., 7: 406-411. DOI: $10.1038 /$ nmat2166

Tang, J., Q. Chen, L. Xu, S. Zhang and L. Feng et al., 2013. Graphene-oxidesilver nanocomposite as a highly effective antibacterial agent with speciesspecific mechanism. ACS Applied Mater. Interfaces, 5: 3867-3874. DOI: 10.1021/am4005495

Tang, L., X. Li, D. Du and C. He, 2012. Fabrication of multilayer films from regenerated cellulose and graphene oxide through layer-by-layer assembly. Progress Nat. Sci.: Mater. Int., 22: 341-346. DOI: 10.1016/j.pnsc.2012.06.005

Terrones, M., A.R. Botello-Méndez, J. Campos-Delgado, F. López-Urías and Y.I. Vega-Cantú et al., 2010. Graphene and graphite nanoribbons: morphology, properties, synthesis, defects and applications. Nano Today, 5: 351-372.

DOI: 10.1016/j.nantod.2010.06.010

Tylianakis, E., G.M. Psofogiannakis and G.E. Froudakis, 2010. Li-doped pillared graphene-oxide: A graphenebased nanostructured material for hydrogen storage. J. Phys. Chem. Lett., 1: 2459-2464. DOI: $10.1021 / \mathrm{jz} 100733 \mathrm{z}$

Wang, H., X. Yuan, Y. Wu, H. Huang and X. Peng et al., 2013. Graphene-based materials: Fabrication, characterization and application for the decontamination of waste water and waste gas and hydrogen storage/generation. Adv. Colloid Interface Sci., 195-196: 19-40.

DOI: $10.1016 /$ j.cis. 2013.03 .009
Wang, L., K. Lee, Y.Y. Sun, M. Lucking and Z. Chen et al., 2009. Graphene oxide as an ideal substrate for hydrogen storage. ACS Nano, 3: 2995-3000. DOI: $10.1021 / \mathrm{nn} 900667 \mathrm{~s}$

Wang, S., M. Yi and Z. Shen, 2016. The effect of surfactants and their concentrations on the liquidexfoliation of graphene. RSC Adv., 6: 56705-56710. DOI: 10.1039/C6RA10933K

Xu, C., A. Cui, Y. Xu and X. Fu, 2013. Graphene oxide$\mathrm{TiO}_{2}$ composite filtration membranes and their potential application for water purification. Carbon, 62: 465-471. DOI: 10.1016/j.carbon.2013.06.035

Yang, W., G. Chen, Z. Shi, C.C. Liu and L. Zhang et al., 2013. Epitaxial growth of single-domain graphene on hexagonal boron nitride. Nat. Mater., 12: 792-797. DOI: $10.1038 /$ nmat3695

Yi, M. and Z. Shen, 2014. Kitchen blender for producing high-quality few-layer graphene. Carbon, 78: 622-626. DOI: 10.1016/j.carbon.2014.07.035

Yi, M. and Z. Shen, 2015. A review on mechanical exfoliation for the scalable production of graphene. J. Mater. Chem. A, 3: 11700-11715. DOI: $10.1039 /$ C5TA00252D

Zhang, D., X. Liu and X. Wang, 2011. Green synthesis of graphene oxide sheets decorated by silver nanoprisms and their anti-bacterial properties. J. Inorganic Biochem., 105: 1181-1186. DOI: 10.1016/j.jinorgbio.2011.05.014

Zhao, L., L. Zhao, Y. Xu, T. Qiu and L. Zhi et al., 2009. Polyaniline electrochromic devices with transparent graphene electrodes. Electrochimica Acta, 55: 491-497. DOI: 10.1016/j.electacta.2009.08.063 